Archives de sciences sociales des religions

155 | juillet-septembre 2011

Le consensus des experts | Inattendus pèlerinages

\title{
Expertise publique sous influence?
}

Rapports publics français et québécois relatifs à l'expression religieuse dans les institutions publiques

David Koussens

\section{(2) OpenEdition}

1 Journals

Édition électronique

URL : http://journals.openedition.org/assr/23306

DOI : $10.4000 /$ assr.23306

ISBN : 978-2-7132-2303-7

ISSN : $1777-5825$

Éditeur

Éditions de l'EHESS

Édition imprimée

Date de publication : 30 septembre 2011

Pagination : 61-79

ISSN : 0335-5985

Référence électronique

David Koussens, "Expertise publique sous influence? », Archives de sciences sociales des religions [En ligne], 155 | juillet-septembre 2011, mis en ligne le 09 novembre 2011, consulté le 21 avril 2019. URL: http://journals.openedition.org/assr/23306; DOI : 10.4000/assr.23306 


\section{David Koussens}

\section{Expertise publique sous influence? \\ Rapports publics français et québécois relatifs à l'expression religieuse dans les institutions publiques}

Moment fort de laïcisation de l'État français, l'adoption de la loi de séparation des Églises et de l'État en 1905 a provoqué une vive émotion, suscitant de nombreux débats autant en France qu'à l'étranger. Si cet événement a contribué à populariser le terme " laïcité », la notoriété publique de celui-ci n'a pas toujours été constante et c'est souvent dans des contextes de tensions que ce "mot qui sent la poudre » (Rivero, 1949 : 137), a été le plus fréquemment employé. Dans la période contemporaine en particulier, la visibilité croissante du religieux a sans doute contribué à l'entrée dans l'usage courant de ce terme qui avait longtemps été l'apanage des spécialistes.

Comme l'a montré J. Baubérot (2009 : 13-14), c'est effectivement en 1989, lors de la première controverse sur le port de signes religieux à l'école publique, que l'emploi de cette notion retrouve une certaine vigueur en France et que de nombreuses contributions défendant une conception d'une laïcité qualifiée de "républicaine" ont été produites. Il en est de même au Québec où, alors que son usage était quasiment inexistant avant 1990, c'est à cette même période que la diversité religieuse devient plus visible et que ce terme fait ses premières apparitions dans les avis et rapports gouvernementaux (Milot, 2009a : 31).

Les conceptions et représentations de la laïcité sont dès lors souvent corrélées avec des événements (parfois qualifiés d' "incidents ») qui recèlent un fort pouvoir symbolique, cela parce qu'ils s'avèrent porteurs d'une importante charge émotive pour ceux qui les vivent (ou pour ceux qui croient les vivre). Ces événements marquent ainsi les esprits et touchent plus directement les consciences que ne le font les multiples aménagements du droit en vertu desquels la laïcité prend pourtant forme dans la gouvernance politique.

Le discours sur la laïcité qui apparaît dans ces contextes peut être qualifié de «laïcité narrative »(Ferrari, 2009), cette notion renvoyant à une forme de laïcité idéalisée qui émerge du discours du pouvoir politique, des organismes institutionnels, mais aussi des acteurs sociaux. Construit social évolutif, la laïcité narrative n'est pas hermétique aux représentations véhiculées dans la société et 
peut sous-tendre des conceptions du bien présumées supérieures qui heurtent certaines valeurs religieuses. Dépourvu de valeur juridique, ce discours n'est pas pour autant dénué de toute force normative (ibid.) En effet, la forme que prennent les aménagements politiques et juridiques de la laïcité dans une société découle de l'interprétation que les gouvernants politiques élaborent à partir de certains de ses principes constitutifs, des principes que la conceptualisation théorique de la laïcité (Milot, 2008) a permis de dégager. Ils correspondent à « l'égalité morale des personnes ou la reconnaissance de la valeur morale égale de chacune d'entre elles ", " la liberté de conscience et de religion ", "la neutralité de l'État à l'égard des religions " ainsi que " la séparation de l'Église et de l'État » (Milot, 2008 : 17-21).

Or, il n'est pas toujours aisé d'harmoniser ces principes constitutifs de la laïcité. Si le compromis peut être difficile à trouver pour les agencer parfaitement, c'est parce que ces principes sont aussi des idéaux. Chacun d'entre eux est peutêtre repérable dans l'histoire des démocraties libérales, mais les interprétations qui en sont faites divergent néanmoins, cela parce qu'ils sont " charg[és] d'un contenu sémantique inévitablement teinté par la hiérarchie des valeurs qui prévaut ici ou là "(Milot, 2009b: 62). Chacun de ces principes peut donc être appréhendé en tant que valeur et l'État laïque va constamment devoir procéder à des arbitrages dans la détermination du poids relatif qu'il leur accorde dans le traitement d'un fait social... et c'est dans ce contexte que la laïcité narrative peut devenir un combustible pour la laïcité juridique et acquérir un poids normatif non négligeable dans la régulation politique et juridique de la diversité religieuse.

C'est cet aspect que je propose d'explorer dans cet article en me penchant sur le discours sur la laïcité qui peut être retracé dans les rapports publics français et québécois relatifs à l'expression des convictions religieuses dans les institutions publiques. Ces rapports constituent une forme de laïcité narrative. Mais parce qu'ils sont commis par des " experts ", ils renvoient à une laïcité narrative que l’on pourrait, à première vue, penser dépassionnée. En outre, parce que leurs conclusions servent souvent de justifications à l'adoption des politiques publiques, ces rapports disposent d'une force normative importante. Dans ce contexte, il est donc utile d'examiner leurs logiques argumentatives afin d'en évaluer la distance effective avec l'émotion et les représentations présentes dans le débat social, mais aussi d'en analyser le poids sur la prise de décision politique. Trois raisons justifient cette analyse.

La première renvoie à la multiplication des rapports relatifs à la régulation de la diversité religieuse au cours des vingt dernières années, cette multiplication traduisant non seulement la complexification du champ religieux contemporain, mais aussi l'importance pour l'État laïque d'en comprendre les manifestations afin de garantir le plus adéquatement la liberté de conscience et de religion de tous les citoyens. Et c'est justement cette garantie, par un État neutre à l'égard des conceptions de la vie bonne, qui nécessite une approche objectivée du champ religieux. 
La deuxième raison justifiant l'analyse tient alors à ce que la multiplication de ces rapports participe d'un processus où l'État ne gère pas directement la charge émotive suscitée par le fait religieux, mais en renvoie le traitement à des commissions ou groupes spécialisés dont l'indépendance et l'expertise sont censées permettre un traitement rationnel d'une question pourtant passionnelle. Ce faisant, il prend en main un problème de nature religieuse - où ce qui est perçu et qualifié comme un problème de nature religieuse - et adopte une politique publique qui apparaît d'autant plus légitime qu'elle procède d'une expertise préalable auprès d'autorités compétentes. La rationalité présumée de ce processus d'expertise semble alors garantir en elle-même la neutralisation de la charge émotive suscitée par le "problème » expertisé.

Pourtant, bien que produit par des experts, le rapport public qui sert d'ancrage à la production juridique n'est évidemment pas toujours uniquement guidé par le souci de garantir des principes de justice, et cela particulièrement quand il est émis dans un contexte de tensions. Il peut alors puiser ses justifications dans des conceptions du bien présentes dans la société qui s'avéreraient défavorables à l'égalité de traitement de toutes les confessions religieuses dans la sphère publique. C'est troisièmement pour cette raison qu'il importe d'expertiser l'expertise, cela pour mieux saisir les déterminants de l'action de l'État dans l'élaboration d'une politique publique ayant trait à la régulation de la diversité religieuse.

Dans ce cadre, l'examen de la mise en œuvre de la neutralité de l'État ouvre des perspectives d'analyse intéressantes. En effet, il a été indiqué que les principes constitutifs de la laïcité ne s'harmonisent pas toujours parfaitement. La neutralité devient donc un indicateur pertinent des aménagements de la laïcité dans une société : l'analyse de sa mise en œuvre permet de remonter aux fondements normatifs qui ont légitimé la prise de décision politique, des fondements que l'on peut notamment retracer dans les différents rapports publics. Les types de neutralité qui y sont décelables sont ainsi révélateurs des positions normatives sousjacentes aux aménagements politiques et juridiques de la laïcité dans une société. Dans ce contexte et afin de mieux cerner les actions par lesquelles l'État va donner un sens aux politiques publiques, l'outil idéal-typique est approprié.

\section{Neutralité confessionnelle et neutralité référentielle ${ }^{1}$}

Selon une approche wébérienne, je propose une modélisation abstraite de deux types de neutralité qui certes n'existent pas tels quels dans la réalité, mais dont on peut néanmoins retrouver des éléments dans les diverses politiques de régulation de la diversité religieuse. Il s'agit donc d'une production idéalisée qui n'a pour objectif que de rendre intelligibles les évolutions de la neutralité. Cette modélisation s'articule autour d'une prise en compte des buts ou des finalités de

1. Ces idéaux-types de la neutralité ont été plus largement développés dans Koussens, 2010. 
l'action de la gouvernance politique au regard d'un système de valeurs particulier, d'une analyse des justifications qui sous-tendent la décision politique ainsi que des effets de cette décision dans la réalité empirique. Ces deux idéaux-types ont été élaborés à partir de l'état de la littérature produite par les auteurs libéraux anglo-saxons. Ils procèdent aussi d'une première observation des diverses postures de l'État dans sa régulation politique et juridique de la diversité religieuse. Pour autant, ces figures ne sont ni des descriptions historiques, ni des positions réellement décelables dans la réalité empirique. Elles ne sont que des outils d'analyse qui permettent justement d'expliquer cette réalité empirique.

Un premier type, la neutralité confessionnelle, correspond à une position de l'État se traduisant par un strict traitement égalitaire des diverses confessions religieuses présentes dans la sphère publique, quitte à ce que certains de ces traitements égalitaires ne soient pas toujours équitables. Concrètement, la neutralité confessionnelle renvoie à une gouvernance politique qui ne peut adopter une position qui avantagerait ou désavantagerait une Église, un culte, une doctrine religieuse ou une famille de pensée en particulier, car ces domaines doivent demeurer ceux de la conscience et du sacré. Le type de la neutralité confessionnelle peut être décelé à partir de plusieurs indicateurs. La politique publique ou la norme juridique :

- n'a pas pour effet direct ou indirect d'octroyer un avantage ou de faire supporter une contrainte sur une confession religieuse ou famille de pensée;

- n'a pas explicitement pour but d'avantager ou de porter ombrage à l'une ou l'autre des confessions religieuses ou familles de pensée;

- n'est pas explicitement justifiée par des considérations favorables ou discriminatoires à l'égard de l'une ou plusieurs des confessions religieuses ou familles de pensée.

Une seconde figure correspond à la neutralité référentielle. Par neutralité référentielle, j'entends la neutralité d'une décision étatique qui n'est pas guidée ou qui ne renvoie pas, implicitement ou explicitement, à une conception du bien spécifique présente dans la société civile en matière religieuse pour définir les principes de régulation de la société à une période déterminée. Il s'agit donc d'un type de neutralité qui ne s'articule que sur des principes de justice. Plusieurs indicateurs permettent de déceler le type de la neutralité référentielle dans la gouvernance politique. La politique publique ou la norme juridique :

- ne se réfère pas explicitement à une conception du bien pour réguler la diversité religieuse ;

- ne se réfère pas implicitement à une conception du bien dans sa régulation de la diversité religieuse ;

- ne se fonde que sur des principes de justice pour réguler la diversité religieuse.

Ces deux idéaux-types peuvent tous deux être décelés dans la laïcité juridique, c'est-à-dire dans les textes officiels, mais aussi dans la laïcité narrative, et dans le cas présent dans les rapports publics. 


\section{Neutralité et rapports publics français}

En France, plusieurs « affaires » relatives à la visibilité du religieux ont ravivé le débat sur l'expression des convictions religieuses dans la sphère publique au début des années 2000. En mai 2003, le député François Baroin a remis au Premier ministre un rapport dans lequel il estimait que face au développement du communautarisme et de l'islamisme, "la laïcité redev[enait] un enjeu politique " (Baroin, 2003). Qualifiant l'avis du Conseil d'État de $1989^{2}$ de «malentendu sur l'analyse de la situation ", le député proposait de réaffirmer les principes de la laïcité pour mieux « relancer la dynamique de l'intégration républicaine » (ibid.) C'est donc dans un contexte social tendu où l'union nationale paraissait menacée (Cherifi, 2005 : 34 ; Thomas, 2008 : 46) que le Président de la République française Jacques Chirac a rouvert le débat sur la laïcité et a confié au haut fonctionnaire Bernard Stasi la présidence d'un groupe de réflexion pour mettre en œuvre une laïcité qui garantirait la cohésion nationale et le respect des différences.

\section{Le rapport de la Commission Stasi (2003)}

D'entrée de jeu, le rapport du 11 décembre 2003 qui découle des travaux de la Commission de réflexion sur l'application du principe de laïcité dans la République (Commission Stasi), rappelle que l'école doit permettre de construire " un destin commun" à tous les Français (2003 : 17). Affirmant que la laïcité de combat, nécessaire à l'établissement de bases stables à la République, n'est plus d'actualité, il souligne que la diversité religieuse à l'école est une richesse. Mais il considère que ce projet est actuellement confronté à un communautarisme croissant porteur d'exclusion.

Le rapport Stasi postule alors que «le principe de neutralité est la première condition de la laïcité " (2003 : 22). Il est le corollaire du principe d'égalité des citoyens et implique que tous les usagers du service public soient traités de la même façon par l'administration. Pour cette raison, « l'administration, soumise au pouvoir politique [doit donner] non seulement toutes les garanties de la neutralité [mais aussi en présenter] les apparences pour que l'usager ne puisse douter de sa neutralité » (ibid.) Le rapport estime alors que « les exigences d'une neutralité absolue sont donc tempérées par les “accommodements raisonnables" permettant à chacun d'exercer sa liberté religieuse »(2003 : 23). Il préconise ainsi

2. Il s'agit de l'avis par lequel le Conseil d'État avait considéré que « le port par les élèves de signes par lesquels ils entendent manifester leur appartenance à une religion n'est pas par lui-même incompatible avec le principe de laïcité " (voir Avis du Conseil d'État portant sur la question de savoir si le port de signes d'appartenance à une communauté religieuse est ou non compatible avec le principe de laïcité, Assemblée générale (section de l'intérieur), 27 novembre 1989, no 346893). 
que " des substituts au porc et le poisson le vendredi [soient] proposés dans le cadre de la restauration collective » sans pour autant que ces mesures n'entravent le fonctionnement normal des services publics (2003: 64). Il indique en effet que si la laïcité implique "l'indépendance du pouvoir politique et des différentes options spirituelles ou religieuses » et donc la neutralité de l'État, ce dernier " ne saurait [pour autant] recouvrir d'un voile d'ignorance le fait spirituel ou religieux ». Une exigence de protection de la liberté de conscience, et plus précisément de garantie de la libre expression des croyances, s'impose donc à lui. Mais le rapport nuance également son propos en précisant que cette exigence n'est pas unilatérale, mais qu'elle est " partagée », notamment dans le cadre scolaire :

"l'exigence laïque demande (...) à chacun un effort sur soi (...) Le citoyen conquiert par la laïcité sa liberté de conscience ; en contrepartie il doit respecter l'espace public que tous peuvent partager (...) Accepter d'adapter l'expression publique de ses particularités confessionnelles et de mettre des bornes à l'affirmation de son identité permet la rencontre de tous dans l'espace public. C'est ce que les Québécois qualifient d'“accommodements raisonnables" " (2003: 16).

Ce faisant, le rapport Stasi s'appuie sur un concept juridique canadien dont il inverse la signification (Koussens, 2008 : 127) ${ }^{3}$, cela principalement pour appuyer une argumentation qui tend vers l'interdiction du port de signes religieux par les élèves dans les écoles publiques. S'il procède ainsi, c'est parce que les prémisses de son raisonnement reposent sur une conception du bien qui n'est pas toujours favorable à l'expression de la liberté de conscience et de religion dans la sphère publique. Plus précisément, dans cette narration, la laïcité est appréhendée comme une "valeur républicaine "(Commission Stasi, 2003 : 10) qui ne "saurait se réduire à la neutralité de l'État. [Selon cette lecture], respect, garantie, exigence, vivre ensemble en sont les principes cardinaux ; ils constituent un ensemble de droit et de devoirs pour l'État, les cultes et les personnes » (2003: 12). Indéniablement, le rapport ne peut donc rejoindre la posture de neutralité référentielle, car il s'appuie clairement sur une conception de la laïcité qui ne renvoie pas qu'aux principes de justice, mais en appelle aussi à une volonté d'émancipation des élèves qui fréquentent l'école publique.

Et pour cette même raison, il ne rejoint qu'indirectement la position de neutralité confessionnelle. Il propose certes que des aménagements ou « accommodements » soient concédés à toutes les confessions dans la sphère publique, mais il se prononce expressément sur la nature de certains signes religieux - la grande

3. Dans une décision de 1985, la Cour suprême du Canada jugeait que « l'obligation dans le cas de la discrimination par suite d'un effet préjudiciable, fondée sur la religion ou la croyance, consiste à prendre des mesures raisonnables pour s'entendre avec le plaignant, à moins que cela ne cause une contrainte excessive (...) il s'agit de prendre les mesures qui peuvent être raisonnables pour s'entendre sans que cela n'entrave indûment l'exploitation de l'entreprise de l'employeur et ne lui impose des frais excessifs ". Voir O’Malley cl Simpson-Sears, [1985] 2 RCS 536. 
croix, la kippa et le voile islamique - qu'il qualifie d'ostensibles et dont il préconise l'interdiction parce qu'ils seraient de nature à "troubler la quiétude de la vie scolaire » $(2003: 41)$.

Le rapport se situe dès lors à mi-chemin entre une figure de laïcité de reconnaissance ${ }^{4}$ et une figure de laïcité séparatiste ${ }^{5}$. Il propose peut-être une meilleure reconnaissance de la diversité religieuse au sein des institutions publiques, cela parce que la laïcité a aussi pour finalité la liberté de conscience, mais il n'accorde pas pour autant la primauté à la justice sociale. Le rapport tend donc vers une figure de laïcité séparatiste, car il accorde une forte prépondérance au principe de séparation. Cette figure renvoie à une logique assimilatrice où la liberté de conscience n'est possible qu'a posteriori, c'est-à-dire après l'émancipation. On y retrace ainsi la dialectique que J. Baubérot (1999: 316) a élaborée entre les figures de "liberté de conscience » et de "liberté de penser»: au nom de la liberté de penser, on peut écorner la liberté de conscience des élèves... mais on l'écorne afin de les émanciper et leur permettre de retrouver a posteriori leur pleine liberté de conscience. Dans cette perspective, la distinction entre l'espace public et celui de la vie privée doit être tangible comme le souligne clairement l'argumentation du rapport Stasi :

« Des pressions s'exercent sur des jeunes filles mineures, pour les contraindre à porter un signe religieux. L'environnement familial et social leur impose parfois des choix qui ne sont pas les leurs. La République ne peut rester sourde an cri de détresse de ces jeunes filles. L'espace scolaire doit rester pour elles un lieu de liberté et d'émancipation $»$ (Commission Stasi, $2003: 58$ ).

L'écho favorable que cette forme de laïcité narrative a trouvé dans la laïcité juridique n'a pas été surprenant. Alors même que la Commission Stasi était en activité, le Président de l'Assemblée nationale J.-L. Debré avait mis en place une Mission d'information sur la question du port de signes religieux à l'école (Debré, 2003) dont les conclusions, rendues publiques le 4 décembre 2003 (soit une semaine avant le dépôt du rapport Stasi) se sont articulées autour d'arguments similaires à ceux du rapport Stasi. Comme en témoignent les débats au Parlement (Koussens, 2010), cette laïcité narrative a fortement influencé les travaux préparatoires à la loi du 15 mars $2004^{6}$, mais elle a également trouvé écho dans la

4. Il s'agit d'une figure de la laïcité inspirée du renouveau de la pensée libérale et qui repose sur la primauté du juste sur le bien et sur le principe de l'autonomie morale des individus (Milot, $2008: 63$ ).

5. Cette figure d'inspiration lockéenne « consiste en une façon de concevoir l'aménagement des principes laïques en mettant l'accent sur une division presque "tangible" entre l'espace de la vie privée et la sphère publique qui concerne l'État et les institutions relevant de sa gouvernance" (Milot, $2008: 46)$.

6. Loi no 2004-228 du 15 mars 2004 encadrant, en application du principe de laïcité, le port de signes ou de tenues manifestant ostensiblement une appartenance religieuse dans les écoles, collèges et lycées publics, JO nº 65 du 17 mars 2004, p. 5190. 
jurisprudence du Conseil d'État qui l'a ensuite mise en application ${ }^{7}$. Légitimée par le droit, cette laïcité narrative a aussi été renforcée par l'adoption de deux autres rapports publics, postérieurs au rapport Stasi, et traitant de l'expression individuelle des convictions religieuses dans l'ensemble des services publics de l'État: le rapport "Rossinot» du 13 septembre 2006 et le rapport du Haut conseil à l'intégration (HCI) de janvier 2007.

\section{Le rapport Rossinot (2006)}

Le rapport Rossinot ne peut être qualifié directement de rapport d'expert. Il est plutôt un rapport politique, commandé par un homme politique - Nicolas Sarkozy en sa qualité de président de l'Union pour un mouvement populaire à un autre homme politique : André Rossinot. Toutefois, il importe de l'aborder, d'une part, parce que son commanditaire exerçait à cette même époque les fonctions de ministre de l'Intérieur et de l'Aménagement du territoire, une fonction impliquant la gestion des affaires cultuelles, d'autre part, parce que ce rapport s'inscrit dans la lignée de celui de la Commission Stasi et trouve de nombreuses correspondances dans un autre rapport produit ultérieurement par le $\mathrm{HCI}^{8}$.

Le rapport Rossinot introduit son propos en postulant que "la laïcité [qui] est d'abord un idéal avant d'être une norme juridique créatrice de droits et d'obligations » (Rossinot, $2006: 3$ ), n'est pas une idée neuve et procède "d'une vision du bien commun forgée par notre histoire et par les choix du peuple français» (idem). Cette vision " exprime l'idée que l'État repose directement sur la volonté des citoyens, sans subordination à aucune autorité supérieure à cette volonté" (idem). Selon cette lecture, la laïcité ne correspond donc pas à un aménagement du politique dans le but de mieux garantir les droits fondamentaux, mais s'apparente à "une valeur, qui ne pourra s'imposer que si les Français y adhèrent massivement» (2006:13). Pour cette raison, le rapport estime que "le rôle des services publics [est] décisif. En effet, c'est principalement au service public qu'il incombe d'œuvrer à la diffusion et à la promotion dans le corps social des valeurs républicaines, et de la laïcité en particulier »(ibid.)

Après avoir diagnostiqué la montée de tensions religieuses dans la sphère publique, ce rapport présente deux types de propositions. Les premières tiennent

7. CE, 5 décembre 2007, M. Chain Singh, n 285394 ; CE, 5 décembre 2007, M. Gurdial Singh, $\mathrm{n}^{\circ} 285395$; CE, 5 décembre 2007, M. Bikramjit Singh, $\mathrm{n}^{\circ} 285396$; CE, 5 décembre 2007, M. et Mme Bessam Ghazal, no 295671.

8. C'est à la même période que le Premier ministre Dominique de Villepin a demandé au HCI d'élaborer une charte sur la laïcité dans les services publics. Il en justifiait alors la nécessité en raison des "préoccupations fortes de nos concitoyens » sur la visibilité du religieux dans la sphère publique. Voir Lettre de saisine du 15 mai 2006 de M. D. de Villepin, Premier ministre à Mme B. Kriegel, Présidente du HCI, http://lesrapports.ladocumentationfrancaise.fr/BRP/ 074000341/0000.pdf 
au renforcement de l'obligation de neutralité des fonctionnaires (2006:49) et à son extension « à tous les agents non statutaires de l'État, des collectivités territoriales et de leurs établissements publics, ainsi qu'aux salariés des entreprises délégataires de services publics et aux collaborateurs du service public » (2006: 48). Les secondes visent à imposer une obligation de neutralité aux usagers des services publics, donc aux citoyens qui fréquentent ces services. De ce point de vue, ces derniers sont tenus de "respecter les principes républicains " dans la sphère publique (2006:24), ce respect prenant corps dans l'abstention de toute demande d'accommodement de nature religieuse auprès de l'administration.

Ce faisant, le rapport Rossinot ne garantit que faiblement la liberté de conscience et de religion. En proposant d'étendre considérablement l'exigence de neutralité dans la sphère publique, il rejoint certes une position de neutralité confessionnelle, mais une position de neutralité confessionnelle qui ne s'avère que minimale, car l'expression du religieux est limitée pour toutes les confessions. L'absence de neutralité référentielle y est par ailleurs explicite. Le rapport puise ses justifications dans une conception du bien (les valeurs de la République) à laquelle il donne une place prépondérante sur les principes de justice. S'inscrivant dans une optique de "laïcité (religion civile) républicaine" (Baubérot, 2009: 16), il rejoint une figure type de laïcité de foi civique d'inspiration rousseauiste, c'est-à-dire une figure où la laïcité peut « [prendre] forme dans un ensemble de valeurs sociales au fondement de la société politique » et s'apparenter ainsi à " une exigence de foi civique » (Milot, 2008 : 59). En contexte français, il s'agit d'une forme de laïcité où les valeurs de la République ne sont pas négociables et auxquelles l'adhésion est un préalable obligatoire au plein exercice de la citoyenneté.

Préconisant en effet l'allégeance de tous les citoyens à des principes civiques "supérieurs ", cette forme de laïcité suspecte les groupes religieux de véhiculer des valeurs inconciliables avec les valeurs républicaines et n'envisage le renforcement du lien politique que par l'adhésion quasi exclusive aux principes républicains. La profession de foi civile évoquée par Rousseau transparaît clairement de l'argumentaire du rapport : "la recherche du consensus ne passe pas par des concessions, mais par l'adhésion du plus grand nombre à des valeurs fortes et assumées " (Rossinot, 2006 : 35). Et selon cette lecture, l'adhésion aux valeurs républicaines n'est pas négociable, car elle est bien " la condition sine qua non de la pérennité du pacte social à long terme » (2006:36). Une conception maximaliste de la neutralité s'imposant dans la sphère publique se profile donc dans ce texte. L'apparence de neutralité ne devrait plus être le simple fait des fonctionnaires, mais aussi celui de tous ceux qui collaborent et participent au service public. Une même expression de cette conception de la laïcité transparaît partiellement dans le rapport du haut conseil à l'intégration (HCI) de janvier 2007. 


\section{Le rapport du haut conseil à l'intégration (2007)}

D'entrée de jeu, le rapport du HCI se dissocie d'une figure de laïcité de foi civique. Il débute en effet en affirmant que « la laïcité représente avant tout une liberté accordée à chacun et non une contrainte imposée à tous » (HCI, 2007 : 191). Selon le rapport, cette laïcité s’inscrit toutefois dans une histoire spécifiquement française au terme de laquelle « l'État républicain, par son prestige et sa neutralité, est devenu l'espace de rencontre et de coexistence de tous les Français » (2007: 194). Mais cet espace serait désormais menacé :

«Dans un contexte de revendications identitaires accrues, le respect et la conciliation des différences s'imposent (...) Or, on ne saurait se résoudre à une segmentation de l'espace public en réponse à la fragmentation des aspirations personnelles. Il y va de la cohésion sociale et nationale » (2007: 196).

S'interrogeant sur «les frontières du service public » (2007: 202), c'est-àdire sur le champ d'exigence de la neutralité de l'État, le rapport en propose aussitôt l'élargissement. Tout comme le rapport Rossinot, il estime que les personnels des organismes délégataires de services publics et les associations privées rémunérées sur des fonds publics devraient être astreints à la même obligation de neutralité que les fonctionnaires de l'État. Il prend par ailleurs le contre-pied de la laïcité juridique relative aux collaborateurs occasionnels du service public ${ }^{9}$ pour demander que ces derniers soient également soumis à une telle exigence de neutralité qu'il souhaite ancrer symboliquement dans une Charte de la lä̈cité dans les services publics, charte diffusée dans l'ensemble des administrations françaises. Concernant les usagers, le rapport se trouve confronté à un dilemme qu'il tente de résoudre en leur imposant une obligation qui ne saurait certes trouver son fondement dans la norme de droit, mais plutôt dans la morale et le respect des valeurs de l'État républicain :

" Au regard des règles contraignantes qui encadrent le comportement des agents publics, le principe de liberté qui est garanti aux usagers est sans aucun doute une illustration majeure de l'ambivalence du principe de laïcité. Pour autant, liberté ne veut pas dire laisser-faire. C'est pourquoi, à défaut d'obligations juridiques systématiques, des obligations morales pèsent sur les usagers, ordonnées autour de quelques règles de conduite raisonnables » (2007: 203-204).

Tout comme dans le rapport Rossinot, la laïcité narrative émergeant des travaux du HCI ne renvoie donc qu'à une posture de neutralité confessionnelle minimale en s'appuyant sur une conception fortement républicaine de la laïcité. Mais cette laïcité narrative s'apparente davantage à la figure de laïcité séparatiste qu'à une laïcité de foi civique. En effet, c'est principalement sur la division entre l'espace de la vie privée et celui qui relève des institutions publiques que l'accent est posé, l’idée étant celle selon laquelle «la laïcité détermine une séparation

9. En effet, le Conseil d'État ne leur impose pas la même obligation de neutralité qu'aux fonctionnaires (voir CE, 27 juillet 2001, Syndicat national pénitentiaire Force ouvrière ; CE, 29 mai 2002, Syndicat national pénitentiaire Force ouvrière). 
inviolable entre les institutions publiques et les symboles religieux ou les signes d'expression religieuse de ceux et celles qui les fréquentent ou y travaillent » (Milot, 2008 : 48). Le HCI conclut ainsi en indiquant que :

« la laïcité bouscule et transcende les communautés naturelles, dont tout humain est issu (...) Elle ne nous appelle pas à renoncer à ce que nous sommes, elle ne nous invite pas à oublier d'où nous venons, à ne pas valoriser telle culture, telles mœurs, telle foi particulière, elle nous appelle à dépasser ensemble toutes les particularités afin de nous rassembler dans un espace plus large, plus neutre et plus ouvert où nous pourrions être associés» (2007: 209).

Suite à ce rapport, le Premier ministre, D. de Villepin, a adopté le 13 avril 2007 une circulaire portant Charte de la laïcité dans les services publics et ayant vocation à être affichée dans l'ensemble des institutions de l'État ${ }^{10}$. Cette charte, qui impose symboliquement une exigence de "neutralité visible » dans les services publics n'a peut-être pas de valeur juridique, mais démontre néanmoins la force de cette forme de laïcité narrative en France. Cet argumentaire a d'ailleurs connu un succès dépassant le champ auquel il devait être cantonné.

Il a d'abord résonné en France et les arguments de la laïcité narrative ont notamment acquis en légitimité en trouvant un point d'appui de taille dans les évolutions récentes de la laïcité juridique. Si l'on retrouve son influence dans la loi de 2004, les récentes décisions du Conseil d'État ${ }^{11}$ se sont également saisies de cette rhétorique républicaine sur l'aménagement du religieux. Cet ajustement du juridique sur le discours sur la laïcité - un ajustement qui n'est toutefois pas absolu, de nombreuses évolutions juridiques ayant aussi permis une meilleure reconnaissance des droits des minorités religieuses (Portier, 2010, 255) - renforce ainsi ce dernier, en le légitimant, mais également en l'alimentant (Koussens, 2009a : 331).

Ces résonances sont également internationales et les arguments de la laïcité narrative française ont ainsi été débattus au Québec dans le cadre de la «crise » des accommodements raisonnables.

\section{Neutralité et rapports publics québécois}

Au Québec, c'est une décision de la Cour suprême du Canada du 2 mars $2006^{12}$ qui a ravivé le débat sur la visibilité du religieux dans la sphère publique. La question qui avait été posée à la Cour était de savoir si l'accommodement consenti par une école québécoise à un jeune sikh, qui consistait à lui permettre d'entrer à l'école avec son kirpan rituel, était valide. S'appuyant sur une tradition

10. Circulaire du Premier ministre du 13 avril 2007 précisant le contenu de la Charte de la laïcité dans les services publics (http://www.fonction publique.gouv.fr/IMG/Circulaire_PM_ 5209_20070413.pdf).

11. Voir note 7.

12. Multani cl Commission scolaire Marguerite-Bourgeoys, [2006] 1 RCS 256, 2006 CSC 6. 
d'interprétation libérale de la Charte canadienne des droits et libertés, la Cour a rappelé la liberté constitutionnelle de conscience et de religion du requérant et, après avoir procédé à un examen in concreto des faits, a indiqué que le principe de l'accommodement raisonnable devait s'imposer à l'institution scolaire.

Cette décision a rapidement suscité la critique et l' " affaire du kirpan » est devenue le catalyseur d'une grande controverse alimentée par les médias. Dans ce contexte, a émergé une importante laïcité narrative, tantôt opposant les principes de justice aux valeurs culturelles défendues dans la société, tantôt essayant au contraire de trouver une dialectique en permettant la conciliation. Face à la crise, la ministre de l'Éducation, du Loisir et du Sport Michelle Courchesne a mis en place un Comité consultatif sur l'intégration et l'accommodement raisonnable en milieu scolaire (Comité Fleury), présidé par un fonctionnaire, Bergman Fleury, dont le mandat était de formuler des recommandations sur la gestion de la diversité dans le domaine de l'éducation.

\section{Le rapport Fleury (2007)}

Le rapport de ce comité composé de fonctionnaires, d'universitaires et de représentants des réseaux scolaires publics et privés a été remis à la ministre le 15 novembre 2007. Alors même que les travaux du comité se sont déroulés pendant la controverse, celui-ci évite de se positionner dans le débat. Son rapport est en effet un document que l'on pourrait qualifier de " technique ", son objectif principal étant de formuler des recommandations pour améliorer concrètement la pratique des accommodements raisonnables en contexte scolaire (Comité Fleury, 2007: 45-46). Une forme de laïcité narrative en ressort néanmoins indirectement, cela parce que le rapport indique que ses recommandations s'inscrivent dans « le cadre juridique en vigueur au Québec, de même que [dans] l'état actuel de la jurisprudence en matière de droits et libertés, notamment l'obligation juridique d'accommodement raisonnable» (2007: 45). Selon cette lecture, il fait siens les aménagements laïques qui procèdent déjà de la jurisprudence de la Cour suprême du Canada, et ce n'est donc qu'indirectement qu'il rejoint les postures de neutralité référentielle et confessionnelle. Il inscrit en effet ses travaux dans un cadre juridique existant qui renvoie à la figure de laïcité de reconnaissance précédemment évoquée. Mais il ne se prononce pas sur ce cadre - ce n'était pas là son mandat - et n'en préconise donc aucun aménagement.

Par ailleurs, si le Comité Fleury a certainement été sensible au contexte dans lequel il a mené ses travaux, il se borne à faire état objectivement des positions présentes dans le débat social, sans prendre position par rapport à celles-ci. Tout en soulignant que «bon nombre de personnes perçoivent certaines demandes d'accommodement raisonnable comme un retour du religieux à l'école qu'ils aimeraient voir réserver à l'espace privé », il ajoute aussi que des "opinions contraires soulignent une conception ouverte de la laïcité des institutions 
publiques qui n'implique pas celle des clientèles » (2007 : 14). En effet, au cours de la controverse, le clivage entre ces positions s'est rapidement creusé et la méfiance a probablement atteint son paroxysme au mois de janvier 2007. Le 14 janvier, le quotidien Le Journal de Montréal publie les résultats d'un sondage selon lequel 59 \% des Québécois s'avoueraient racistes ${ }^{13}$. Le 27 janvier, le quotidien La Presse fait état d'un texte, adopté par la municipalité d'Hérouxville, présentant les normes de vie auxquelles devrait se conformer tout immigrant qui souhaiterait s'établir dans cette commune. Dans ce contexte de tensions, le Premier ministre du Québec, Jean Charest, a annoncé en février 2007 la création d'une Commission de consultation sur les pratiques d'accommodement reliées aux différences culturelles dirigée par les universitaires Gérard Bouchard et Charles Taylor (Commission Bouchard-Taylor) avec le mandat de mener une consultation publique à l'échelle du Québec et de recueillir les mémoires des individus, groupes, organismes ou institutions qui voulaient faire entendre leur voix sur la question des accommodements raisonnables.

\section{Le rapport Bouchard-Taylor (2008)}

Refusant de restreindre ses travaux au seul champ des accommodements, la Commission Bouchard-Taylor, composée très majoritairement d'universitaires, a étendu son mandat à l'ensemble des interrogations suscitées par "le modèle d'intégration socioculturelle instauré au Québec » (Bouchard et Taylor, 2007: 3) et notamment à la question de la laïcité. Dans son rapport final, remis au Premier ministre le 22 mai 2008, elle rappelle ainsi que ses recommandations ont pour objectif de "préciser et d'officialiser les grandes orientations de la société québécoise quant aux rapports interculturels " (Bouchard et Taylor, 2008 : 249), des orientations au nombre desquelles elle inclue la laïcité.

Le rapport précise que la laïcité ne peut se résumer à des formules simples comme « séparation des Églises et de l'État » ou "sortie de la religion de l'espace public » même si ces formules révèlent une part de vérité (2008 : 135). Il reprend alors les quatre principes constitutifs - que nous avons évoqués plus haut - aux fondements de la laïcité et indique que les modalités d'articulation de ceux-ci permettent de déceler deux modèles principaux de laïcités, qu'il qualifie de régimes de laïcités "intégrales " ou "rigides » et de régimes de laïcités plus « souples» ou « ouvertes» (2008: 137).

Selon cette lecture, le premier de ces régimes "permet une restriction plus grande du libre exercice de la religion au nom d'une certaine interprétation de la neutralité de l'État et de la séparation des pouvoirs politiques et religieux » (ibid.) D'une part, la laïcité « rigide » est justifiée par l'invocation d'un principe

13. Le Journal de Montréal, "Constat troublant », 14 janvier 2007, http://www.canoe.com/ infos/dossiers/archives/2007/01/20070114-191421.html 
d'émancipation des individus de la religion. Le rapport rejette cependant ce principe, son application s'avérant problématique dans une société plurielle comme le Québec et révélant, en outre, une forme de laïcité non neutre à l'égard des «raisons profondes qui animent les individus » (ibid.) D'autre part, ce régime repose sur la promotion de l'intégration civique ou «allégeance à une identité civique commune " (ibid.) À nouveau, le rapport estime qu'une telle promotion serait inadéquate au Québec où «le développement d'un sentiment d'appartenance et d'identification dans une société (...) passe davantage par une "reconnaissance raisonnable" des différences que par leur relégation stricte à la sphère privée " (ibid.)

Le rapport préconise alors le ralliement au second régime de laïcité, un régime de laïcité « ouverte » qui caractérise les relations entretenues entre les Églises et l'État au Québec, et «défend un modèle axé sur la protection de la liberté de conscience et de religion, ainsi qu'une conception de la neutralité étatique plus souple» $(2008,137-140)$. On notera ici que la forme de laïcité narrative qui ressort de ce rapport était déjà prégnante dans plusieurs travaux d'organismes institutionnels québécois produits avant la controverse par le Comité sur les affaires religieuses de ministère de l'Éducation, des Loisirs et des Sports (2003) et le Conseil des relations interculturelles (2004). S'inscrivant ainsi dans la lignée d'une réflexion entamée dans un contexte moins passionné, le rapport BouchardTaylor indique que c'est bien en raison du choix du modèle de laïcité pour lequel le Québec a opté qu'il faut poursuivre les pratiques d'accommodements raisonnables (Bouchard et Taylor, 2008: 159). Il en rappelle néanmoins les balises déjà établies, notamment le principe de la «contrainte excessive » (2008 : $163)$, mais refuse toutefois d'y inclure les valeurs culturelles présentes dans la société :

"Les conventions sociales ou les valeurs coutumières ne sont pas illégitimes pour autant, mais elles ne sauraient justifier l'usage du pouvoir coercitif de l'État contre des personnes qui ne s'y conforment pas; par exemple, on ne peut pas demander à l'État d'interdire le port des signes religieux visibles en invoquant une norme sociale (...) comme unique justification » $(2008: 164)$.

Selon l'argumentation du rapport, les pratiques d'accommodements raisonnables trouvent leurs justifications, mais aussi leurs limites, dans le respect des droits fondamentaux. Cette logique fondée sur des principes de justice est valable pour les usagers des services de l'administration, mais elle l'est aussi pour les fonctionnaires auxquels on ne saurait interdire le port de signes religieux. Le rapport indique en effet que :

"l'interdiction pour les agents de l'État de porter des signes religieux a un double prix, à savoir la restriction de a) la liberté de conscience et de religion des personnes visées et, éventuellement, $b$ ) l'égalité dans l'accès aux emplois de la fonction publique et parapublique» $(2008: 149)$.

Dans cette optique, l'apparence de neutralité des institutions publiques peut certainement constituer une garantie de la confiance que lui accordent les 
citoyens, mais ne saurait cependant justifier l'interdiction générale du port de signes religieux pour les employés de l'État (ibid.) Ce faisant, le rapport propose de présumer a priori de la neutralité de ces derniers pour n'en évaluer l'effectivité qu'a posteriori au regard de leur action :

«ce qui importe (...) est que les agents de l'État fassent preuve d'impartialité dans l'exercice de leurs fonctions. Un employé de l'État doit chercher à accomplir la mission attribuée par le législateur à l'institution qu'il sert ; ses actes ne doivent lui être dictés ni par sa foi ni par ses croyances philosophiques, mais bien par la volonté de réaliser les finalités associées au poste qu'il occupe » (2008:149).

Toutefois, le rapport ne recommande pas d'autoriser le port de signes religieux aux employés de l'État dans toutes circonstances et procède à une double distinction. D'abord, il préconise que ce port soit limité s'il engendre une " contrainte excessive » à l'égard du fonctionnement du service, c'est-à-dire s'il peut «entraver l'accomplissement de la fonction occupée » (2008: 150). Il indique que :

" une enseignante ne pourrait par exemple revêtir une burqa ou un niqab en classe et s'acquitter adéquatement de sa tâche d'enseignante. D'une part, l'enseignement passe par définition par la communication et le recouvrement du visage et du corps exclut la communication non verbale. D'autre part, l'une des missions de l'enseignante est de contribuer au développement de la sociabilité de l'élève » (2008: 150).

Sans se prononcer sur la symbolique du signe religieux en question, le rapport n'évalue donc la possibilité de son port qu'au regard du fonctionnement du service. Ensuite, il distingue selon la nature des fonctions exercées par les fonctionnaires et précise que seuls ceux d'entre eux qui détiennent un pouvoir de sanction ou de coercition peuvent légitimement se voir imposer une obligation de réserve absolue en matière religieuse (2008 : 151). Dès lors, le rapport propose que le port de signe religieux "soit interdit aux magistrats et procureurs de la Couronne, aux policiers, aux gardiens de prison [et] aux présidents et viceprésidents de l'Assemblée nationale ", les deux derniers devant être contraints par cette obligation en raison de leur fonction de représentation de la souveraineté du peuple (2008: 271).

Cette argumentation rejoint ainsi une position de neutralité confessionnelle que l'on peut qualifier d'intégrale. En effet, en ne s'immisçant pas dans les " convictions de conscience » dont sont porteurs les individus, le rapport évite l'écueil de l'interprétation et garantit largement l'expression de la liberté de conscience et de religion pour toutes les confessions. Il rejoint également la posture de neutralité référentielle parce que, sans pour autant valoriser la diversité, il ne propose pas de projet de transformation des comportements sociaux des individus. Il ne se fonde en effet que sur la reconnaissance des droits fondamentaux et promeut, ce faisant, une forme de laïcité de reconnaissance.

On remarquera que, contrairement au contexte français, la laïcité narrative procédant des rapports publics québécois n'a pas servi de combustible aux développements de la laïcité juridique; cela probablement parce que ce discours sur 
la laïcité était déjà en adéquation avec les aménagements juridiques par lesquels l'État régule la diversité religieuse, des aménagements qui se caractérisent par une vraie constance depuis ces vingt dernières années et qui ont en outre été confortés par les plus récentes décisions des juridictions canadienne et québécoise. Bien que jamais affirmée dans une norme juridique, la neutralité de l'État y émane pourtant clairement du travail d'interprétation du droit par les tribunaux. Dans ce processus, elle est appréhendée comme une obligation qui ne s'impose qu'au politique, et non aux citoyens comme dans le cas français. Pour cette raison, le droit est jusqu'à présent resté hermétique aux arguments d'une laïcité narrative de type séparatiste émergeant pourtant dans le débat social et dont certains partis politiques - dont le Parti Québécois - se font les porte-voix (Koussens, 2009b), pour se cantonner à son rôle d'arbitre et favoriser l'aménagement d'une réelle laïcité de reconnaissance.

\section{Conclusion}

Alors que la laïcité ne peut être considérée comme une valeur commune partagée par les citoyens d'une société déterminée, les aménagements laïques s'articulent pourtant dans des systèmes de valeurs propres à chaque société. Les principes qui ressortent de la conceptualisation théorique de la laïcité sont de " grands" principes. Mais ils sont aussi de " grands » idéaux dont le contenu sémantique et la charge symbolique dépendent des arbitrages auxquels procèdent les pouvoirs publics.

Ces arbitrages sont rarement dictés par le législateur lui-même. Quand celuici est muet en contexte québécois, les termes "laïcité » et «neutralité de l'État " n'étant pas affirmés dans les textes de loi, il s'avère particulièrement laconique dans le cadre français, ne se contentant que d'énoncer ces principes en fixant le cadre général dans lequel ils devront être opérationnalisés. Pour cette raison, si Gérard de la Pradelle (1979 : 44) indique que le «sens final [de la règle de droit] est fourni par le juge [et qu'ultimement] la loi est ce que ce dernier affirme trouver dans les textes ", on peut ajouter que les aménagements laïques sont le fruit d'un travail qui ne se fait pas seulement en aval de la production législative, c'est-à-dire par les tribunaux qui donnent sens à la norme de droit, mais aussi en amont, dans les travaux qui président à l'élaboration de cette norme de droit et en fournissent les justifications.

C'est à ce titre que les rapports d'experts acquièrent toute leur importance, cela parce qu'ils interprètent eux aussi les valeurs de la société pour donner sens aux principes aux fondements de la laïcité. Or ce faisant, il importe qu'ils ne se nourrissent pas de ces valeurs et fassent preuve de neutralité pour ne pas entretenir un discours partisan sur la laïcité qui pourrait, à terme, trouver écho dans la norme de droit. Le danger du " passage de ces récits de l'implicite à l'explicite » (Ferrari, 2009) serait en effet celui d'une «laïcité durcie, produit [de ce] récit 
capable de plier le droit à un projet de clôture sociétale face aux périls du pluralisme » (ibid.) Légitimé par l'expertise, le droit risquerait dès lors de ne plus dépasser le conflit, mais de s'en faire l'allégorie.

\section{David KOUSSENS \\ European University Institute (Italie) \\ David.Koussens@eui.eu}

\section{Bibliographie}

Baroin François, 2003, Pour une nouvelle laïcité. Rapport, http://www.voltairenet.org/ rubrique 506.html

Baubérot Jean, 1999, "Laïcité, sectes, société », in Champion F., Cohen M., (éds.), Sectes et démocratie, Paris, Éditions du Seuil, pp. 314-330.

-, 2009, "Liberté, laïcité, diversité - la France multiculturelle », in Eid P., Bosset P., Milot M., Lebel-Grenier S. (éds.), Appartenances religieuses, appartenances citoyennes. Un équilibre en tension, Québec, Presses de l’Université Laval, pp. 13-26.

Cherifi Hanifa, 2005, « Application de la loi du 15 mars 2004 », Hommes et migrations, novembre-décembre, pp. 33-47.

Comité consultatif sur l'intégration et l'accommodement raisonnable en milieu scolaire, 2007, Une école québécoise inclusive : dialogue, valeurs et repères communs, http:// www.mels.gouv.qc.ca/sections/accommodement/pdf/RapportAccRaisonnable.pdf

Comité sur les affaires religieuses, Ministère de l'Éducation, du Loisir et du Sport, 2003, Rites et symboles religieux à l'école. Défis éducatifs de la diversité, Québec, Gouvernement du Québec.

Commission de consultation sur les pratiques d'accommodements reliés aux différences culturelles, 2007, Accommodements et différences. Vers un terrain d'entente : la parole aux citoyens. Document de consultation, http://www.accommodements.qc.ca/ documentation/document-consultation.pdf

Commission de consultation sur les pratiques d'accommodements reliés aux différences culturelles, 2008, Fonder l'avenir. Le temps de la conciliation, http://www. accommodements.qc.ca/documentation/rapports/rapport-final-integral-fr.pdf

Commission de réflexion sur l'application du principe de laïcité dans la République, 2003, Rapport remis au président de la République, http://lesrapports.ladocumentationfrancaise. fr/BRP/034000725/0000.pdf

Conseil des relations interculturelles, 2004, Laïcité et diversité religieuse : l'approche québécoise, disponible en ligne sur http://www.conseilinterculturel.gouv.qc.ca

DeBRÉ Jean-Louis, 2003, Rapport fait au nom de la mission d'information sur la question $d u$ port de signes religieux à l'école, http://www.assemblee-nationale.fr/12/rapports/ r1275-t1.asp\#P736_183922

FERRARI Alessandro, 2009, "De la politique à la technique : laïcité narrative et laïcité du droit. Pour une comparaison France/Italie ", in Basdevant-Gaudemet B., Jankowiak F., (éds.), Le droit ecclésiastique de la fin $d u \mathrm{XVIII}^{e}$ au milieu $d u \mathrm{XX}^{e}$ siècle en Europe, Leuven, Peeters, pp. 333-345. 
Haut Conseil à l'intégration, 2007, Projet de charte de la laïcité dans les services publics. Avis à Monsieur le Premier ministre, http://lesrapports.ladocumentationfrancaise.fr/ BRP/074000341/0000.pdf

Koussens David, 2008, "Le port de signes religieux dans les écoles québécoises et françaises. Accommodements (dé)raisonnables ou interdiction (dé)raisonnée », Globe. Revue internationale d'études québécoises, 10-2, pp. 115-131.

-, 2009a, «Sous l'affaire de la burqa... quel visage de la laïcité française », Sociologie et sociétés, 41-2, pp. 327-347.

-, 2009b, "Comment les partis politiques québécois se représentent-ils la laicité ? ", Diversité urbaine, 9-1, pp. 27-44.

-, 2010, "L'État français et l'expression des convictions religieuses : entre neutralité confessionnelle et neutralité référentielle ", Politique et sociétés, 29-3, pp. 39-60.

La Pradelle de Géraud, 1979, L’homme juridique, Grenoble, Les Presses Universitaires de Grenoble, coll. "Critique du droit».

Milot Micheline, 2008, La laïcité, Ottawa, Novalis, coll. « 25 questions ».

-, 2009a, "L'émergence de la notion de laïcité au Québec. Résistances, polysémie et instrumentalisation », in Eid P., Bosset P., Milot M., Lebel-Grenier S., (éds.), Appartenances religieuses, appartenances citoyennes. Un équilibre en tension, Québec, Les Presses de l'Université Laval, pp. 29-73.

-, 2009b, «Laïcité au Canada. Liberté de conscience et exigence d'égalité ", Archives de sciences sociales des religions, 146, pp. 61-80.

PORTIER Philippe, 2010, "Modernités plurielles" ? Une approche longitudinale des modèles nationaux de régulation du croire dans les démocraties occidentales ", in Milot M., Portier P., Willaime J.-P., (éds.), Pluralisme religieux et citoyenneté, Rennes, Presses universitaires de Rennes, pp. 241-271.

RIVERo Jean, 1949, "La notion juridique de laïcité », Recueil Dalloz, XXXIII, pp. 137140.

RossinOt André, 2006, La laïcité dans les services publics. Rapport, http://www.islamlaicite. org/IMG/pdf/Rapport_Rossinot.pdf

Thomas Carole, 2008, «Interdiction du port du voile à l'école. Pratiques journalistiques et légitimation d'une solution législative à la française ", Politique et Sociétés, 7-2, pp. 41-71.

\section{Résumé}

À partir d'une modélisation abstraite des types de neutralité décelables dans la gouvernance publique, cet article propose une analyse des postures de neutralité que l'on peut retracer dans les rapports publics français et québécois relatifs à l'expression individuelle des convictions religieuses dans les institutions publiques. Il en explore les logiques argumentatives afin d'en évaluer la distance effective avec l'émotion et les représentations présentes dans le débat social du moment, mais aussi d'en analyser le poids sur la prise de décision politique.

Mots-clés : laïcité, neutralité, rapports publics, France, Québec. 


\section{Abstract}

Based on an abstract modeling of types of neutrality that are detectable in public governance, this article proposes to analyze the maturation of these types of neutrality in the French and Québecois publics reports relative to the individual expression of beliefs in public institutions. It explores these report's argumentations in order to evaluate, on the one hand, its distance with emotions and dominant representations present in social debate, in the second hand, their weight on the adoption of public policies.

Key words: laicity, neutrality, public reports, France, Québec.

\section{Resumen}

A partir de una modelización abstracta de los tipos de neutralidad subyacentes a la gobernanza pública, este artículo propone un análisis de las posiciones de neutralidad que se pueden reconstituir en las relaciones públicas entre Francia y Quebec relativas a la expresión individual de las convicciones religiosas en las instituciones públicas. El artículo explora las lógicas argumentativas para evaluar la distancia efectiva con la emoción y las representaciones presentes en el debate social del momento, pero también para analizar su peso sobre la toma de decisiones politicas.

Palabras clave: laicidad, neutralidad, relaciones públicas, Francia, Quebec. 
